\title{
COUNTING PERFECT COLOURINGS OF PLANE REGULAR TILINGS
}

\author{
DIRK FRETTLÖH
}

\begin{abstract}
A first step in investigating colour symmetries of periodic and nonperiodic patterns is determining the number of colours which allow perfect colourings of the pattern under consideration. A perfect colouring is one where each symmetry of the uncoloured pattern induces a global permutation of the colours. Two cases are distinguished: Either perfect colourings with respect to all symmetries, or with respect to orientation preserving symmetries only (no reflections). For the important class of colourings of regular tilings (and some Laves tilings) of the Euclidean or hyperbolic plane, this mainly combinatorial question is addressed here using group theoretical methods.
\end{abstract}

\section{Introduction}

The study of colour symmetries of crystallographic patterns in Euclidean space is a classical topic, see for instance [6], [10] and references therein. The discovery of quasicrystals inspired interest in colour symmetries of further (quasi-)crystallographic patterns, see [1], [8], 9]. A very recent development is the study of colour symmetries of regular tilings in the hyperbolic plane [4. For several reasons, the focus of studying colour symmetries frequently lies on perfect colourings (for a precise definition, see below). For instance, a perfect colouring yields a simple algebraic relation between the symmetry group $G$ of the uncoloured pattern and the symmetry group $K$ of the coloured pattern: The factor group $G / K$ is a permutation group. Thus, basic questions to address in the study of colour symmetries are determining the possible numbers of colours for which a perfect colouring exists, counting the different perfect colourings with this number of colours, and study algebraic properties of the colour groups. This article answers the first two questions for perfect colourings of regular (and certain Laves tilings), be it spherical tilings, or tilings in the Euclidean plane, or in the hyperbolic plane. The case of Euclidean tilings is well known for long, see [10] for a good survey. The case of regular tilings of the sphere is somehow trivial. Therefore, the focus of this article lies on counting perfect colourings of hyperbolic regular (and certain hyperbolic Laves) tilings. The main result is a method to count these perfect colourings based on counting certain subgroups of Coxeter groups. This is outlined in Section 3 with respect to the full symmetry group of regular tilings, and in Section 4 with respect to the rotation group of regular tilings. As an application, some concrete results are listed in Table 1 and 2 .

\section{Colourings, regular tilings and Laves tilings}

Let $\mathbb{X}$ be either the Euclidean plane $\mathbb{R}^{2}$, or the hyperbolic plane $\mathbb{H}^{2}$, or the sphere $\mathbb{S}^{2}$. An isometry $f: \mathbb{X} \rightarrow \mathbb{X}$ is called a symmetry of some set $X \subset \mathbb{X}$, if $f(X)=X$. Here, $X$ can be nearly anything living in $\mathbb{X}$, but it is fine to think of $X$ as some point set, or some tiling. The set of all symmetries of $X$ form a group, the symmetry group of $X$. A symmetry is called orientationpreserving, if it is a translation, or a rotation, or a product of those. The subgroup of the symmetry group of $X$ containing all orientation-preserving symmetries is called the rotation group of $X$. In the remainder of this section, the symmetry group of some set $X$ is always denoted by $G$, as well as the rotation group of $X$. This should not lead to confusion, everything works for both cases.

In the sequel, the symmetric group of order $n$ (all permutations on $n$ numbers) is denoted by $\mathcal{S}_{n}$. A $k$-colouring $(X, c)$ of $X$ is a surjective map $c: X \rightarrow\{1, \ldots, k\}$. We are mainly interested in colourings, where each $h \in G$ acts as a global permutation of colours. That is, we consider those $h \in G$ where

$$
\forall x, y \in X: c(x)=c(y) \Rightarrow c(h(x))=c(h(y))
$$


Following [6, we call a pair $(h, \pi)$ (where $\left.h \in G, \pi \in \mathcal{S}_{k}\right)$ a colour symmetry of $(X, c)$, if $\pi(c(x))=$ $c(h(x))$ for all $x \in X$. The group of all colour symmetries

$$
C(X)=\left\{(h, \pi) \mid h \in G, \pi \in \mathcal{S}_{k}: c(h(x))=\pi(c(x)) \text { for all } x \in X\right\}
$$

is called the colour symmetry group of $X$. For convenience, let $H:=\{h \mid(h, \pi) \in C(X)\}$. Since each $h \in H$ determines a unique permutation $\pi_{h}, H$ is isomorphic to $C(X)$. The map $h \mapsto \pi_{h}$ is a group homomorphism, which can be seen as follows: Let $i \in\{1, \ldots, k\}$. Then, for all $x \in X$ with $c(x)=i$ holds

$$
\pi_{g h}(i)=\pi_{g h}(c(x))=c(g h(x))=c(g(h(x)))=\pi_{g}\left(c(h(x))=\pi_{g} \pi_{h}(c(x))=\pi_{g} \pi_{h}(i),\right.
$$

hence $\pi_{g h}=\pi_{g} \pi_{h}$. A colouring is called perfect, if $H=G$, or equivalently: if every symmetry of $X$ is a colour symmetry of $(X, c)$.

Example: Consider an infinite checkerboard (Figure 1). It can be regarded as a 2-colouring of the canonical square tiling of the plane. The symmetry group $G$ of the uncoloured tiling is the Coxeter group generated by reflections in the lines $a, b, c$. A reflection $R_{a}$ in $a$ interchanges the colours of the checkerboard: All black squares are mapped to white squares and vice versa. Thus, $R_{a} \in H, \pi_{R_{a}}=(1,2)$. The reflections $R_{b}$ and $R_{c}$ both fix the colours, therefore $R_{b}, R_{c} \in H$, $\pi_{R_{b}}=\pi_{R_{c}}=\mathrm{id}$. All three generators of $G$ are also elements of $H$, thus $G=H$, and the colouring is perfect.

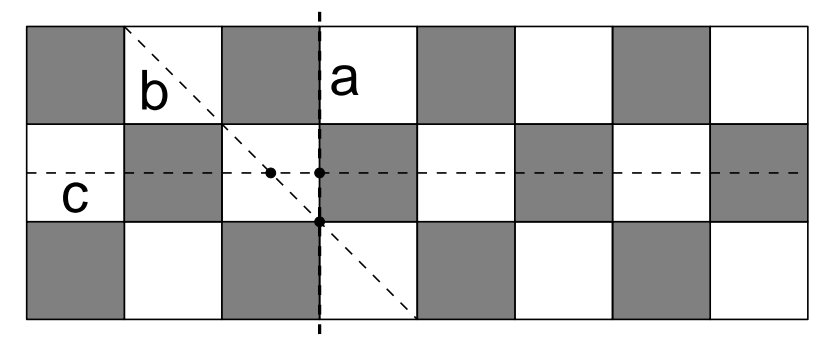

Figure 1. Part of an infinite checkerboard. A reflection in the line $a$ interchanges the colours globally. Reflections in the lines $b$ or $c$ fix the colours.

A tiling of $\mathbb{X}$ is a collection of compact sets $T_{1}, T_{2}, \ldots$ which cover $\mathbb{X}$ (that is, the union of all $T_{i}$ is $\mathbb{X}$ ) and which do not overlap (that is, the intersection of the interiors of any two sets $T_{i} \neq T_{j}$ are empty). The sets $T_{i}$ are called tiles. A tiling of $\mathbb{X}$ is called regular, if all tiles are regular polygons, and all vertex figures are regular polygons. (The vertex figure of a vertex $x$ in a tiling is the convex hull of all midpoints of the edges emanating from $x$.) Following $\left[6\right.$, a tiling of $\mathbb{R}^{2}$ or $\mathbb{H}^{2}$ is called Laves tiling, if all tiles are congruent, and all vertex figures are regular polygons. For a thorough survey on regular tilings and Laves tilings see [3] and [6].

Any ordered pair of integers $(p, q)$, where $p, q \geq 3$, defines a regular tiling by regular $p$-gons, where $q$ tiles meet at each vertex. Following [6], such a tiling is denoted by $\left(p^{q}\right)$. If $\frac{1}{p}+\frac{1}{q}>\frac{1}{2}$, then $\left(p^{q}\right)$ is a regular tiling of the sphere (which can be regarded as a regular polytope in $\mathbb{R}^{3}$ ). If $\frac{1}{p}+\frac{1}{q}=\frac{1}{2}$, then $\left(p^{q}\right)$ is a regular tiling of the Euclidean plane $\mathbb{R}^{2}$. If $\frac{1}{p}+\frac{1}{q}<\frac{1}{2}$, then $\left(p^{q}\right)$ is a regular tiling of the hyperbolic plane $\mathbb{H}^{2}$. In $\mathbb{R}^{2}$ there are only three different regular tilings and eleven different Laves tilings (including the three regular ones, see [6]). In $\mathbb{S}^{2}$ there are five regular tilings (corresponding to the five platonic solids) and eight Laves tilings (including the former five, see [3]). In contrast, there are infinitely many regular tilings in $\mathbb{H}^{2}$, as well as Laves tilings. Here we consider the following Laves tilings only: Each regular tiling $\left(p^{q}\right)$ gives rise to a Laves tiling [p.q.p.q] (if $p<q$, otherwise we write [q.p.q.p], in order to make the notation unique) by joining the centres of each $p$-gons with each of its vertices by line segments. Consider these line segment as the edges of a new tiling. Then this tiling will be a tiling by congruent quadrilaterals, with either $p$ or $q$ tiles at each vertex. (Note that for the regular square tiling in $\mathbb{R}^{1}$, this constructions yields again a regular square tiling $[4.4 .4 .4]=\left(4^{4}\right)$; whereas the regular hexagon tiling $\left(6^{3}\right)$ yields a Laves tiling [3.6.3.6] by $60^{\circ}$ rhombi.) 
The symmetry group of $\left(p^{q}\right)$ is a Coxeter group $G_{p, q}$ :

$$
G_{p, q}=\left\langle a, b, c \mid a^{2}=b^{2}=c^{2}=(a b)^{q}=(a c)^{2}=(b c)^{p}=1\right\rangle .
$$

$G_{p, q}$ is also the symmetry group of both $\left(q^{p}\right)$ and [p.q.p.q]. The latter fact is the reason, why we can easily include these certain Laves tilings in our study, where other Laves tilings require further effort. A fundamental domain of $G_{p, q}$ is an orthogonal triangle $F_{p, q}$ with angles $\frac{\pi}{2}, \frac{\pi}{p}, \frac{\pi}{q}$. Then, $a$ (b,c, resp.) denotes the reflection in the line spanned by the edge of $F$ which is opposite to the angle $\frac{\pi}{p}\left(\frac{\pi}{2}, \frac{\pi}{q}\right.$, resp. $)$.

Left coset colouring: (compare 4]) The orbit of $F:=F_{p, q}$ under $G_{p, q}$ is a tiling $T_{p, q}$ of $\mathbb{X}$ by triangles $f F$, where $f$ runs through $G_{p, q}$. In particular, this gives a bijection between elements of $G_{p, q}$ and elements of $T_{p, q}$. Therefore, any subgroup $S \subseteq G_{p, q}$ of index $k$ induces a $k$-colouring of $T_{p, q}$ in the following way: Set $c(f F)=1$ for each $f \in S$, and set $c(f F)=i$, if $f$ is element of the $i$-th left coset $S_{i}$ of $S=S_{1}(1 \leq i \leq k)$. In the next section, we deduce colourings of regular tilings $\left(p^{q}\right)$ from these colourings of $T_{p, q}$.

This construction is taken from [4, where it is given in a more general setup. Here it reduces to the above, since the stabiliser of $F$ in $G_{p, q}$ is trivial. However, the following lemma is true in a more general context than that of this article.

Lemma 2.1. All colourings of a tiling obtained by the left coset colouring method are perfect.

Proof: Let $S$ be the subgroup of $G_{p, q}$ inducing the colouring of $T_{p, q}$. Each tile in $T_{p, q}$ is of the form $f F$, where $f \in G_{p, q}$. We need to show that for each symmetry $g \in G_{p, q}$ holds: $c(f F)=c\left(f^{\prime} F\right)$ implies $c(g f F)=c\left(g f^{\prime} F\right)$. By the left coset construction, $c(f F)=c\left(f^{\prime} F\right)$ is equivalent to $f$ and $f^{\prime}$ being in the same left coset. That is, $f^{-1} f^{\prime} \in S$. Consequently, $(g f)^{-1}\left(g f^{\prime}\right)=f^{-1} g^{-1} g f^{\prime}=f^{-1} f^{\prime} \in S$, and the claim follows.

\section{Counting colourings with respect to the symmetry group}

In this section we present a method to count colour symmetry groups for any regular tiling or Laves tiling in $\mathbb{R}^{2}$ or $\mathbb{H}^{2}$ or $\mathbb{S}^{2}$ with respect to the entire symmetry group of the tiling. We should emphasise that different colourings may possess the same colour symmetry group (see [10, $\S 8]$ ). Colourings with the same colour symmetry group are strongly related, but not necessarily congruent. So what we count here are not different colourings, but different colour symmetry groups, where different means non-conjugate. This is the usual approach, compare [2, [10. In general, the question how two different colourings with the same colour symmetry group are related can be quite hard to answer.

Note that $T_{p, q}$ can be obtained from $\left(p^{q}\right)$ by dividing each $p$-gon into $2 p$ triangles congruent to $F$. In the following, we are interested in colourings of $\left(p^{q}\right)$ (respectively [p.q.p.q], respectively $\left(q^{p}\right)$ ) rather than colourings of $T_{p, q}$. To obtain a colouring (of $\left(p^{q}\right)$, say) we just need to consider those colourings of $T_{p, q}$, where all $2 p$ triangles forming a $p$-gon possess the same colour.

Lemma 3.1. In a perfect colouring of a regular tiling $\left(p^{q}\right)$ or a Laves tiling [p.q.p.q], all colours occur with the same frequency.

Proof: The symmetry group $G_{p, q}$ acts transitively on the tiles. Thus, for all pairs of colours $(i, j)$, there is $f_{i j} \in G_{p, q}$ mapping some tile of colour $i$ to some tile of colour $j$. Since the colouring is perfect, $f_{i j}$ maps the entire colour class $c^{-1}(i)$ to the colour class $c^{-1}(j)$. Since $f_{i j}$ is an isometry, $c^{-1}(i)$ and $c^{-1}(j)$ have the same frequency.

The last result is not true in general for perfect colourings of other tilings.

Lemma 3.2. Let $S$ be a subgroup of $G_{p, q}$ of index $k$. $S$ induces a perfect $k$-colouring of $\left(q^{p}\right)$, if and only if $a, b \in S$. $S$ induces a perfect $k$-colouring of $\left(p^{q}\right)$, if and only if $b, c \in S$. S induces a perfect $k$-colouring of [p.q.p.q], if and only if $a, c \in S$.

Proof: We provide the proof for the case $\left(q^{p}\right)$. The other cases are completely analogous.

One direction ('if') is clear from the construction and from Lemma 2.1 above. 
For the other direction, let $\left(\left(q^{p}\right), c\right)$ be a perfect $k$-colouring. Let $K_{i}=\left\{f \in G_{p, q} \mid c(f F)=i\right\}$. Without loss of generality, let id $\in K_{1}$. Then

$$
\pi\left(K_{1}\right)=\left\{\pi_{f} \mid f \in K_{1}\right\}=\left\{\pi_{f} \mid \pi_{f}(1)=1\right\}
$$

is a subgroup of the symmetric group $\mathcal{S}_{k}$. Since $f \mapsto \pi_{f}$ is a group homomorphism, $K_{1}$ is a subgroup of $G_{p, q}$. By the last lemma, all colour classes have the same frequency, thus the index of $K_{1}$ in $G_{p, q}$ is $k$. Since the entire $q$-gon $F \cup a F \cup a b F \cup a b a F \ldots$ has colour 1 , one has $a, a b, a b a, \ldots \in K_{1}$. Finally, let $i$ and $f F \in K_{i}$ be fixed. For each $g F \in K_{i}$ holds $c(g F)=i$, hence $\pi_{g}(1)=i$, and $\left(\pi_{g}\right)^{-1}(i)=\pi_{g^{-1}}(i)=1$. It follows

$$
c\left(g^{-1} f F\right)=\pi_{g^{-1}} c(f F)=\pi_{g^{-1}} \pi_{f}(c(F))=\pi_{g^{-1}} \pi_{f}(1)=\pi_{g^{-1}}(i)=1 .
$$

Therefore, $g^{-1} f F \in K_{1}$ for all $g \in K_{i}$, thus $K_{i}=f K_{1}$ is a coset of $K_{1}$.

By the last result, we obtain all perfect $k$-colourings of $\left(q^{p}\right)$ (respectively $[p . q . p . q]$, respectively $\left(p^{q}\right)$ ) by listing the subgroups of $G_{p^{q}}$ which contain $a, b$ (respectively $a, c$, respectively $b, c$ ). This can be carried out by GAP [5], for instance. See Table 1 for some examples. Subgroups are identified if they are conjugate in $G_{p, q}$.

\begin{tabular}{|c|l|}
\hline$\left(7^{3}\right)$ & $1,8,15,22,24,30,36^{2}, 44,50^{5}, \ldots$ \\
\hline$[3.7 .3 .7]$ & $1,9,15,21,28^{2}, 30,35^{2}, 36,37,42^{5}, 49^{8}, 50^{3}, \ldots$ \\
\hline$\left(3^{7}\right)$ & $1,22,28^{5}, 37,42^{4}, 44,49^{7}, 50^{3}, \ldots$ \\
\hline \hline$\left(8^{3}\right)$ & $1,3,6,12,17,21^{4}, 24,25^{5}, 27^{3}, 29^{4}, 31^{4}, 33^{6}, 37^{6}, 39^{8}, \ldots$ \\
\hline$[3.8 .3 .8]$ & $1,3,6,12^{3}, 17,18,21^{4}, 24^{15}, 25^{5}, 27^{3}, 28^{4}, 29^{4}, 30^{7}, \ldots$ \\
\hline$\left(3^{8}\right)$ & $1,2,4,8,10^{2}, 12,14,16^{2}, 18,20^{4}, 24^{3}, 25^{5}, 26,28^{12}, 29,30^{2}, \ldots$ \\
\hline \hline$\left(5^{4}\right)$ & $1,2,6,11,12,16^{2}, 21^{3}, 22^{5}, 24,26^{9}, 28, \ldots$ \\
\hline$[4.5 .4 .5]$ & $1,5^{2}, 10^{5}, 11,15^{7}, 20^{22}, 21^{3}, 22^{4}, 25^{27}, 26^{4}, 27^{3}, 30^{63}, \ldots$ \\
\hline$\left(4^{5}\right)$ & $1,5^{2}, 10^{4}, 11,15^{7}, 16,20^{9}, 21^{3}, 22,25^{27}, 26,27^{3}, 30^{38}, \ldots$ \\
\hline
\end{tabular}

TABLE 1. The list of the first possible values $k$ for perfect $k$-colourings of some hyperbolic regular and Laves tilings. An upper index $n \geq 2$ means that there are $n$ non-conjugate colour symmetry groups for this value. No upper index means there is exactly one colour symmetry group for this value, up to conjugacy.

The procedure is quite general. In principle, all colour symmetries of all plane regular tilings can be obtained in this manner, be it hyperbolic, Euclidean or spherical tilings. Regular spherical tilings in $\mathbb{S}^{2}$ can be regarded as regular polytopes in $\mathbb{R}^{3}$. For instance, the cube $\left(4^{3}\right)$ allows perfect colourings with 1,3 and 6 colours, each one unique, up to permutation of colours. The icosahedron $\left(3^{5}\right)$ allows unique perfect colourings with 1, 10 and 20 colours. However, this method has a drawback: Practically, computing with finitely presented groups is not efficient. Therefore, there is for each $\left(p^{q}\right)$ a limit on the number of colours to be considered. Table 1 lists the first values of the number of perfect colourings of some regular and some related Laves tilings in $\mathbb{H}^{2}$.

\section{Counting colourings with respect to the rotation group}

In the last section colourings were considered which are perfect with respect to the entire symmetry group $G_{p, q}$. A slightly weaker, but still important concept is to consider colourings which are perfect with respect to the rotation group. These include of course all colourings of the last section, but also many pairs of enantiomorphic colourings, where one is obtained from the other by a reflection. Let us denote the rotation group of the regular tiling $\left(p^{q}\right)$ by $\bar{G}_{p, q}$. It is also the symmetry group of both $\left(q^{p}\right)$ and $[p . q . p . q]$. It is a subgroup of $G_{p, q}$ of index 2 . $\bar{G}_{p, q}$ is spanned in $G_{p, q}$ by $a b, a c$, compare (11). (With $a b$ and $a c$ it contains also $b c$. It does not matter which two generators out of $\{a b, a c, b c\}$ are chosen.) Now, analogously to Lemma 3.2, certain subgroups of $\bar{G}_{p, q}$ induce colourings of $\left(p^{q}\right)$ or $[p . q . p . q]$ which are perfect with respect to $\bar{G}_{p, q}$.

Lemma 4.1. Let $S$ be a subgroup of $\bar{G}_{p, q}$ of index $k$. S induces a perfect $k$-colouring of $\left(q^{p}\right)$ if and only if $a b \in S$. $S$ induces a perfect $k$-colouring of [p.q.p.q] if and only if ac $\in S$. $S$ induces a perfect $k$-colouring of $\left(p^{q}\right)$ if and only if bc $\in S$. 
Note that 'perfect' is to be read here as 'perfect with respect to the rotation group'. The proof is completely analogous to the one of Lemma 3.2 using the fact, that $F \cup a F$ and $F \cup b F$ are both fundamental domains of $\bar{G}_{p, q}$.

Counting the perfect colourings is slightly more subtle as in the last section. Again subgroups are identified if they are conjugate. But two subgroups have to be identified not only if they are conjugate in $\bar{G}_{p, q}$, but also if they are conjugate in $G_{p, q}$. This requires some programming in GAP, and the computations become even harder. (The program code can be requested from the author.) For instance, there are 119 subgroups of index 20 in $\bar{G}_{5,4}$ inducing perfect colourings of [4.5.4.5]. These have to be checked pairwise for being conjugate in $G_{5,4}$. Without further refinements, this takes weeks using GAP on a usual PC, which is the reason why this particular value has not been included in Table 2, The groups in Table 1 are contained in Table 2

\begin{tabular}{|c|l|}
\hline$\left(7^{3}\right)$ & $1,8,9,15^{2}, 22^{7}, 24, \ldots$ \\
\hline$[3.7 .3 .7]$ & $1,7,9,14^{6}, 15^{2}, 21^{5}, 22^{7}, \ldots$ \\
\hline$\left(3^{7}\right)$ & $1,7,8,14^{6}, 21^{2}, 22^{7}, \ldots$ \\
\hline \hline$\left(8^{3}\right)$ & $1,3,6,9,10,12,13^{2}, 15,17^{5}, 18^{5}, 19^{5}, \ldots$ \\
\hline$[3.8 .3 .8]$ & $1,3,4,6,8^{3}, 9,10^{2}, 12^{4}, 13^{2}, 15,16^{9}, 17^{5}, 18^{12}, \ldots$ \\
\hline$\left(3^{8}\right)$ & $1,2,4,8^{4}, 10^{3}, 12,13^{2}, 14^{2}, 16^{12}, 17^{5}, 18,19^{5}, \ldots$ \\
\hline \hline$\left(5^{4}\right)$ & $1,2,6^{2}, 11^{3}, 12^{6}, 16^{12}, 17^{4}, \ldots$ \\
\hline$[4.5 .4 .5]$ & $1,5^{2}, 6,10^{9}, 11^{3}, 12^{4}, 15^{15}, 16^{10}, 17^{4}, \ldots$ \\
\hline$\left(4^{5}\right)$ & $1,5^{2}, 6,10^{6}, 11^{3}, 15^{15}, 16^{2}, 17^{4}, \ldots$ \\
\hline
\end{tabular}

TABLE 2. The list of the first possible values $k$ of perfect $k$-colourings of some hyperbolic regular and Laves tilings, using the same scheme as Table 1. The groups in Table 1 are contained in this table.

\section{Conclusion}

The left coset colouring method of [4] to obtain perfect colourings of certain tilings was used to obtain perfect colourings of regular tilings $\left(p^{q}\right)$ (or Laves tilings [p.q.p.q]). The use of Coxeter groups $G_{p, q}$ allowed for a bijection between perfect colourings of these tilings and subgroups of $G_{p, q}$. This allowed us to deduce the numbers $k$ for which a $k$-colouring exists, and their multiplicities (up to conjugacy of the colour symmetry groups). As an application, we provide a list of these numbers $k$ together with their multiplicities for some hyperbolic regular and Laves tilings.

\section{Acknowledgements}

It is a pleasure to thank the second referee for very valuable remarks. This work was supported by the German Research Council (DFG) within the CRC 701.

\section{REFERENCES}

[1] M. Baake: Combinatorial aspects of colour symmetries, J. Phys. A: Math. Gen. 30 (1997) 2687-2698; mp_arc/02-323.

[2] M. Baake, U. Grimm: Bravais colourings of planar modules with $N$-fold symmetry, Z. Krist. 219 (2004) 72-80, math.C0/0301021.

[3] H.S.M. Coxeter: Regular Polytopes, Metheun \& Co Ltd., London (1948).

[4] M.L.A.N. de las Peñas, R.P. Felix, G.R. Laigo: Colorings of hyperbolic plane crystallographic patterns, $Z$. Krist. 221 (2006) 665-672.

[5] The GAP Group, GAP - Groups, Algorithms, and Programming, Version 4.4.10 (2007); http://www.gap-system.org.

[6] B. Grünbaum, G.C. Shephard: Tilings and patterns, Freeman, New York (1987).

[7] J.E. Humphreys: Reflection Groups and Coxeter Groups, Cambridge University Press (1990).

[8] R. Lifshitz: Theory of color symmetry for periodic and quasiperiodic crystals, Rev. Mod. Phys. 69 (1997) $1181-1218$

[9] R. V. Moody, J. Patera: Coloring of quasi-crystals, Can. J. Phys. 72 (1994) 442-452.

[10] R. L. E. Schwarzenberger: Colour symmetry, Bull. London Math. Soc. 16 (1984) 209-240. 
Fakultät für Mathematik, Universität Bielefeld, Postfach 100131, 33501 Bielefeld, Germany

E-mail address: dirk.frettloeh@math.uni-bielefeld.de

$U R L:$ http://www.math.uni-bielefeld.de/baake/frettloe 